Draft VERSion OCTOBER 24, 2018

Preprint typeset using $\mathrm{LAT}_{\mathrm{E} X} \mathrm{X}$ style emulateapj v. 11/12/01

\title{
X-MAS2: STUDY SYSTEMATICS ON THE ICM METALLICITY MEASUREMENTS
}

\author{
E. Rasia ${ }^{1,2,3}$, P. Mazzotta ${ }^{4,5}$, H. Bourdin ${ }^{4}$, S. Borgani ${ }^{6,7,8}$, \\ L. Tornatore $^{9}$, S. Ettori ${ }^{10}$, K. Dolag ${ }^{11}$, L. Moscardini $^{12}$ \\ rasia@umich.edu \\ Draft version October 24, 2018
}

\begin{abstract}
The X-ray measurements of the intra-cluster medium metallicity are becoming more and more frequent due to the availability of powerful X-ray telescope with excellent spatial and spectral resolutions. The information which can be extracted from the measurements of the $\alpha$-elements, like Oxygen, Magnesium and Silicon with respect to the Iron abundance is extremely important to better understand the stellar formation and its evolutionary history. In this paper we investigate possible source of bias or systematic effects connected to the plasma physics when recovering metal abundances from X-ray spectra. To do this we analyze 6 simulated galaxy clusters processed through the new version of our X-ray MAp Simulator (X-MAS), which allows to create mock XMM-Newton EPIC MOS1 and MOS2 observations. By comparing the spectroscopic results inferred by the X-ray spectra to the expected values directly obtained from the original simulation we find that: $i$ ) the Iron is recovered with high accuracy for both hot $(T>3 \mathrm{keV})$ and cold $(T<2 \mathrm{keV})$ systems; at intermediate temperatures, however, we find a systematic overestimate which depends inversely on the number counts; ii) Oxygen is well recovered in cold clusters, while in hot systems the X-ray measurement may overestimate the true value by a factor up to 2-3; iii) Being a weak line, the measurement of Magnesium is always difficult; despite of this, for cold systems (i.e. with $T<2 \mathrm{keV}$ ) we do not find any systematic behavior, while for very hot systems (i.e. with $T>5 \mathrm{keV}$ ) the spectroscopic measurement may strongly overestimate the true value up to a factor of $4 ; i v$ ) Silicon is well recovered for all the clusters in our sample. We investigate in detail the nature of the systematic effects and biases found performing XSPEC simulations. We conclude that they are mainly connected with the multi-temperature nature of the projected observed spectra and to the intrinsic limitation of the XMM-Newton-EPIC spectral resolution that does not always allow to disentangle among the emission lines produced by different elements.
\end{abstract}

Subject headings: cosmology: miscellaneous - methods: numerical - galaxies: clusters: general - X-ray: spectrum - hydrodynamics.

\section{INTRODUCTION}

Measuring the metallicity of the intra-cluster medium (ICM) represents a unique means towards a unified description of the thermodynamical properties of the diffuse gas and of the past history of star formation within the population of cluster galaxies (e.g., Renzini et al. 1993). The total mass in metals is directly related to the number of supernovae (SNe) which exploded in the past. Furthermore, measuring the relative abundance of Iron with respect to $\alpha$-elements provides information on the relative fraction of type II and type Ia SN and, therefore, on the shape of the initial mass function (IMF) for star forma- tion. Finally, the redshift evolution of the metal content of the ICM reflects the rate at which metals are released in the diffuse medium. This, in turn is directly related to the star formation history.

From an observational point of view, measurements of the global content of different metal species within the ICM have been first made possible with the advent of the ASCA satellite (e.g., Fukazawa et al. 1994; Mushotzky et al. 1996; Fukazawa et al. 1998; Finoguenov et al. 1999; Finoguenov \& Ponman 1999; Fukazawa et al. 2000; Finoguenov et al. 2000; Finoguenov \& Jones 2000;

${ }^{1}$ Department of Physics, University of Michigan, 450 Church St., Ann Arbor, MI 48109-1120, USA

2 Dipartimento di Astronomia, Università di Bologna, via Ranzani 1, I-40127, Bologna, Italy

${ }^{3}$ Chandra Fellow

${ }^{4}$ Dipartimento di Fisica, Università di Roma Tor Vergata, via della Ricerca Scientifica 1,

I-00133, Roma, Italy

${ }^{5}$ Harvard-Smithsonian Centre for Astrophysics, 60 Garden Street, Cambridge, MA 02138, USA

${ }^{6}$ Dipartimento di Astronomia, Università di Trieste, via Tiepolo 11, I-34131, Trieste, Italy

7 INAF - Osservatorio Astronomico di Trieste, via Tiepolo 11, I-34131, Trieste, Italy

${ }^{8}$ INFN - National Institute for Nuclear Physics, I-34100, Trieste, Italy

9 SISSA/International School for Advanced Studies, via Beirut 4, I-34100, Trieste, Italy

10 INAF, Osservatorio Astronomico di Bologna, via Ranzani 1, I-40127, Bologna, Italy

11 Max-Planck-Institut für Astrophysik, Karl-Schwarzschild Strasse 1, D-85748, Garching bei München, Germany

12 INFN, Sezione di Bologna, viale Berti Pichat 6/2, I-40127 Bologna, Italy 
Matsushita et al. 2000; Finoguenov et al. 2001). Based on a collection of ASCA data for a fairly large number of clusters, Baumgartner et al. (2005) determined the relation between the global ICM content of different metal species and the temperature. At the same time, data from the Beppo-SAX satellite allowed to perform a detailed study of the spatial distribution of Iron (De Grandi \& Molendi 2001). This study showed that significant negative metallicity gradients are present in "cooling flow" clusters, with a central metallicity spike associated to the brightest cluster galaxy (De Grandi et al. 2004). More recently, the excellent spatial resolution of Chandra has allowed to obtain Iron metallicity profiles (Ettori et al. 2002; Buote et al. 2003; Dupke \& White 2003; Humphrev et al. 2006) and maps (Sanders et al. 2004; Sanders \& Fabian 2006) for a significant number of clusters. Furthermore, the large collecting area of XMM-Newton is now allowing to study in detail the abundances of several elements, thereby providing a test-bed for different models of SNe yields de Plaa et al. 2006, 2007; Böhringer et al. 2005, and reference therein), and to largely improve the description of the metal content associated to cluster cool cores (Molendi \& Gastaldello 2001; Tamura et al. 2001; Böhringer et al. 2002; Finoguenov et al. 2002; Gastaldello \& Molendi 2002; Sakelliou et al. 2002; Matsushita et al. 2003; Böhringer et al. 2004; Tamura et al. 2004; Werner et al. 2006; Matsushita et al. 2007a). Chandra and XMM-Newton allowed to analyze in detail the Iron bias already noticed with $A S C A$ (Buote 2000a). This bias is arising when fitting with a single temperature model a plasma characterized by a temperature gradient or by a multi-temperature structure with the colder component below $1 \mathrm{keV}$ (Molendi \& Gastaldello 2001; Buote et al. 2003). More recently, the Suzaku telescope is providing accurate detections of Oxygen and Magnesium lines and has already provided measurements of the distribution of $\alpha$-elements in the Fornax cluster (Matsushita et al. 2007b) and in Abell 1060 (Sato et al. 2007) out to fairly large cluster-centric distances. Finally, archival studies based on Chandra and XMM-Newton observations are now providing information on the evolution of the global Iron metal content out to $z \sim 1.3$ and show a decrease of about 50 per cent of the ICM metallicity from the present time to that redshift (Balestra et al. 2007; Maughan et al. 2007).

From the theoretical point of view, a number of methods have been developed to describe the ICM enrichment associated with the cosmological hierarchical build up of galaxy clusters. De Lucia et al. (2004), Nagashima et al. (2005) and Bertone et al. (2007) studied the ICM enrichment by coupling $N$-body simulations to semi-analytical models (SAMs) of galaxy formation. This approach, although quite flexible to explore the space of parameters relevant for star formation and galaxy evolution, does not provide detailed information on the spatial distribution of metals within clusters and on the role of gasdynamical processes (e.g., ram-pressure stripping, turbulent diffusion, etc.) in distributing metals. In order to overcome these limitations, Cora (2006) developed a hybrid technique, which is based on applying SAMs to non-radiative hydrodynamical simulations. Another approach is that of simulating clusters with hydrodynami- cal codes, which included a treatment of specific effects, such as galactic winds (Schindler et al. 2005), ram pressure stripping (Domainko et al. 2006) and active galactic nuclei (Moll et al. 2007), although coupled with simplified descriptions of star formation. Finally, cosmological hydrodynamical simulations of galaxy clusters have been presented in the last few years, in which the production of metals is self-consistently related to the process of gas cooling and star formation, by accounting in detail for the contribution from different stellar populations and the corresponding life-times (Lia et al. 2002; Valdarnini| 2003; Tornatore et al. 2004; Romeo et al. 2006; Tornatore et al. 2007).

These numerical descriptions of the ICM enrichment have reached a good enough accuracy, in terms of physical processes included and resolution, so as to allow a detailed comparison with observational data to be performed. It is however clear that for this comparison to be meaningful, one has to be sure to understand observational biases, related to the instrumental response of the $\mathrm{X}$-ray detectors onboard of the different satellites. A typical example of such biases in the study of the ICM is represented by the measure of the X-ray temperature. Mazzotta et al. (2004) showed that the combination of a complex thermal structure of the ICM and of the finite energy band, within which $\mathrm{X}$-ray spectra are measured, can bias low the value of the spectroscopic temperature (see also Vikhlinin 2006). Therefore, it is worth asking whether similar biases also affect the determination of the ICM metallicity through the fitting of the $\mathrm{X}$-ray spectrum with single-temperature and single-metallicity plasma models.

In this paper we address the issue of the biases in the $\mathrm{X}$-ray measurement of the ICM metallicity by performing mock observations of hydrodynamical simulations of galaxy clusters, which include a detailed description of the process of chemical enrichment and follow the production of different chemical species (Tornatore et al. 2007). To this purpose, we generate XMM-Newton observations of both EPIC-MOS1 and EPIC-MOS2 cameras, performed with a new version of the X-ray MAp Simulator (X-MAS, Gardini et al. 2004) package, which now also includes the contribution from metal lines in the generation of the $\mathrm{X}_{-}^{-}$ ray spectra. Taking advantage of this tool, we can carefully check for the presence of systematics in the X-ray measurement of the metallicity, also by distinguishing the behavior of Iron and of $\alpha$-elements, when observations are performed at the spectral resolution of XMM-Newton. In particularly we investigated the biases related only to the physics of the plasma. The biases related to the uncertainties on the calibration and/or on the background and/or on plasma model are out of the scope of this paper. As such, this paper mainly focusses on quantifying the possible presence of these systematics and understand their relation with the complex thermal and chemical structure of the ICM. We defer to a forthcoming analysis a comparison between results of hydrodynamical simulations of ICM enrichment and observational data (Fabjan et al. in preparation).

The plan of the paper is as follows. The new version of our X-ray MAp Simulator, X-MAS2, will be presented in Sect. 2. The characteristics of the hydrodynamical simulations are described in Sect. 3. The procedure followed for 
the X-ray analysis is presented in Sect. 4, Our results are described in Sect. 5, where we also provide a critical discussion about the effect of observing X-ray spectra with the typical XMM-Newton energy resolution (Sect. [5.6). These results are discussed in Sect. 6, while the main conclusions of our analysis are summarized in Sect. 7 .

All errors quoted are at $1 \sigma$ level (68.3 per cent level of confidence for one interesting parameter).

\section{X-MAS2}

The numerical code X-MAS Gardini et al. 2004) produces mock Chandra and XMM-Newton observations starting from outputs of hydro-N-body simulations of galaxy clusters. The software is composed by two main parts: the first section computes the emissivity of each gas particle contained in the selected field of view and projects it into the sky plane; the second one convolutes the flux reaching the telescope using the response of a given detector. One of the main characteristics of X-MAS is that it is able to create the events file containing all the spectral information for each "incoming simulated photon". Moreover it properly reproduces the spatial features and the characteristics of the Chandra and XMMNewton detectors. Furthermore the header of the final event file stores all the fundamental keywords common to true observed files. In this way the application of the routines and analysis programs commonly used by observers is straightforward. In previous work the code has been used only in the Chandra ACIS-S3 mode to address several issues, like the difference between the emission-weighted and spectroscopic temperature definitions Gardini et al. 2004), the validity of the spectroscopic-like formula proposed by Mazzotta et al. (2004) and the possible sources of bias in the X-ray estimates of cluster masses (Rasia et al. 2005, 2006). To do that the ICM metallicity has been kept fixed at 0.3 solar (assuming Anders \& Grevesse 1989) and the emissivity has been computed simply adopting a MEKAL model (Mewe et al. 1985, 1986; Liedahl et al. 1995). For the purpose of this paper a much more detailed analysis of the metal content of the ICM is required. Therefore, X-MAS has been subject to a substantial upgrading which affected both the first and second parts.

\subsection{First part: implementing the metallicity}

We refer to Gardini et al. (2004) for a detailed derivation of the incoming photons flux, $F_{\nu}^{\gamma}$. Here we report its final expression as a function of redshift $z$, luminosity distance $d_{L}$, Emission Measure $E M \equiv \int_{V} n_{e} n_{H} d V^{\prime}$ (where the integrals of the electron, $n_{e}$, and hydrogen, $n_{H}$, densities are computed over the volume $V$ ) and power coefficient $P_{\nu(1+z)}^{\gamma}(T, Z)$ :

$$
F_{\nu}^{\gamma}=\frac{(1+z)^{2}}{4 \pi d_{L}^{2}} E M P_{\nu(1+z)}^{\gamma}(T, Z) .
$$

Notice that the power coefficient is a function of both temperature and metallicity. To take this latter into account, the metal content is split in different elements and their contribution is computed separately.

Thus, the total power coefficient per each particle is given by:

$$
\begin{aligned}
P_{\nu(1+z)}^{\gamma}\left(T, Z_{\text {tot }}\right)= & P_{\nu(1+z)}^{\gamma}(T, H)+ \\
& \Sigma_{i} m\left(Z_{i}\right)\left[P_{\nu(1+z)}^{\gamma}\left(T, Z_{i}\right)-P_{\nu(1+z)}^{\gamma}(T, H)\right]
\end{aligned}
$$

where the sum is over each chemical element, $Z_{i}$, considered in the simulation (He included). The power coefficients are computed assuming a single-temperature VMEKAL model, where we fix to 1 solar value the contribution of each element, namely $\mathrm{He}, \mathrm{C}, \mathrm{N}, \mathrm{O}, \mathrm{Mg}, \mathrm{Si}$ and $\mathrm{Fe}$. The spectrum computed by VMEKAL for each element, $P_{\nu(1+z)}^{\gamma}\left(T, Z_{i}\right)$, will be the sum of the continuum, given by the power coefficient corresponding to hydrogen, $P_{\nu(1+z)}^{\gamma}(T, H)$, plus the contribution of the different elements. The factor $m\left(Z_{i}\right)$ represents the weight equal to the $Z_{i}$-element mass in units of its Solar values (defined using the abundances reported in Anders \& Grevesse 1989). Hereafter we will compute the emissivity by using a MEKAL model, nevertheless the code includes a flag to switch to the APEC model (and corresponding VAPEC for multi-metal elements treatment, see Smith et al. 2001) whenever desired.

In the new version of the simulator we have also modified the routine based on the SPH kernel (Monaghan \& Lattanzio 1985) to distribute the particle emissivity, and assign the interesting quantities to the projected pixels. The change consists in computing the integration over the area through the products of two integrals along the two directions of the plane, where each integral has been analytically calculated at priori. This trick significantly speeds up the computational time.

As in the previous version of X-MAS, we also include the effect of the Galactic HI absorption. Chosen a value of the column density $N_{H}$, we multiply the flux in each energy channel by an absorption coefficient computed adopting the WABS model (Morrison \& McCammon 1983).

\subsection{Second part: simulating EPIC observations}

Once the projected flux has been obtained by the first unit of X-MAS, the second unit takes care of the simulation of cluster "observations". This second part has been implemented to simulate XMM-Newton observations made with the EPIC camera. This is done by generating a set of photons, given the incoming ICM and chosen an exposure time. These are then applied to a ray-tracing procedure designed to mimic the main characteristics of the telescope optical paths and detector responses. (See also Bourdin et al. 2004).

The incoming set of photons $\{p(k, l, e)\}$, having position on mirrors $[k, l]$ and individual energy $e$, is generated from random realizations of the total energy expected assuming a given ICM flux $F_{\nu}^{\gamma}$, a telescope area $A$, and an exposure time $t$. At this aim, we project the energies $t \times A \times F_{\nu}^{\gamma}$ along the line of sight, and we store the obtained quantity in a three-dimensional array (where two dimensions represent the position on the mirrors and the third one is for energy). Assuming a Poisson statistics, we can finally associate a discrete number of photons to the resulting total energies. Initially we consider an array having angular and spectral resolutions of $\Delta r=3 \operatorname{arcsec}$ and $\Delta e=20$ $\mathrm{eV}$, which are below the expected performances of the $X M M-N e w t o n$-EPIC instruments.

The ray-tracing procedure emulating the EPIC observations comprises a set of filtering and redistribution functions of the photon energies and positions across the detector planes. Information about the different instrumental effects are coming from ground and on-flight calibra- 
Rasia et al.

tions, and essentially provided in the XMM-Newton Current Calibration Files (CCF) and EPIC Redistribution Matrix Files (RMF). The main steps of our procedure are as follows:

- redistribution of the photon coordinates, $[k, l]$, according to the instrument Point Spread Functions (PSF), given as a function of the position on mirrors and energy of the incidental photons;

- rejection of part of the incoming photons due to the spatially dependent cross-section of mirrors, the socalled "vignetting";

- suppression of photons falling outside CCDs or within dead pixels;

- rejection of part of the incoming photons due to energy dependent effective area of mirrors, filter absorption, and quantum efficiency of detectors;

- redistribution of photon energies $e$, according to the detector response;

- addition of particles induced false detections, with random drawing performed following the relative energy spectrum expected for a given exposure time.

We finally obtain a list of events, $\{e v(k, l, e)\}$, corresponding to photon detections - but also including some false ones due to particles -, with registered positions on the detector plane, $[k, l]$, and energies, $e$.

\section{THE SIMULATED CLUSTERS}

The six simulated clusters analyzed in this paper have been selected from a larger ensemble of simulations of 19 clusters presented by Saro et al. (2006); we refer to that paper for more details. These clusters are extracted from a parent DM-only simulation (Yoshida et al. 2001) with a box size of $479 \mathrm{~h}^{-1} \mathrm{Mpc}$ of a flat $\Lambda \mathrm{CDM}$ model with $\Omega_{0 \mathrm{~m}}=0.3$ for the present matter density parameter, $h=0.7$ for the Hubble constant in units of $100 \mathrm{~km}$ $\mathrm{s}^{-1} \mathrm{Mpc}^{-1}, \sigma_{8}=0.9$ for the r.m.s. fluctuation within a top-hat sphere of $8 h^{-1} \mathrm{Mpc}$ radius and $\Omega_{0 \mathrm{~b}}=0.04$ for the baryon density parameter. Mass resolution is increased inside the interesting regions by using the Zoomed Initial Condition (ZIC) technique proposed by Tormen et al. (1997). Besides the low-frequency modes, which were taken from the initial conditions of the parent simulation, the contribution of the newly sampled high-frequency modes was also added. Once initial conditions are created, we split particles in the high-resolution region into a DM and a gas component, whose mass ratio is set to reproduce the assumed cosmic baryon fraction. In the high-resolution region, the masses of the DM and gas particles are set to $m_{\mathrm{DM}}=1.13 \times 10^{9} h^{-1} \mathrm{M}_{\odot}$ and $m_{\text {gas }}=1.7 \times 10^{8} h^{-1} \mathrm{M}_{\odot}$, respectively. The Plummer-equivalent softening length for the gravitational force is set to $\epsilon_{\mathrm{Pl}}=5 h^{-1} \mathrm{kpc}$, kept fixed in physical units from $z=5$ to $z=0$, while being $\epsilon_{\mathrm{Pl}}=30 h^{-1} \mathrm{kpc}$ in comoving units at higher redshifts.

The simulations have been carried out with a version of the GADGET-2 code 13 (Springel 2005), which

13 http://www.MPA-Garching.MPG.DE/gadget/ includes a detailed treatment of chemical enrichment from stellar evolution (Tornatore et al. 2004, 2007). GADGET-2 is a parallel Tree+SPH code with fully adaptive time-stepping, which includes an integration scheme which explicitly conserves energy and entropy (Springel \& Hernquist 2002), the effect of a uniform and evolving UV background (Haardt \& Madau 1996), star formation from a multiphase interstellar medium and a prescription for galactic winds triggered by SN explosions (see Springel \& Hernquist 2003 for a detailed description), and a numerical scheme to suppress artificial viscosity far from the shock regions (see Dolag et al. 2005). In the original version of the code, energy feedback and global metallicity were produced only by SNII under the instantaneousrecycling approximation (IRA). We have suitably modified the simulation code, so as to correctly account for the lifetimes of different stellar populations, to follow metal production from both SNIa, SNII, as well as from low and intermediate mass stars, while self-consistently introducing the dependence of the cooling function on metallicity (Sutherland \& Dopita 1993). A detailed description of the implementation of these algorithms is presented in detail by Tornatore et al. (2007). The simulations analyzed here assume the power-law shape for initial stellar mass function, as proposed by Salpeter (1955) and galactic ejects with a speed of $500 \mathrm{~km} \mathrm{~s}^{-1}$.

The main global properties of the simulated clusters are reported in Table 1. In the sample there are two big objects with mass (computed inside the radius $R_{200}$ which comprises an over-density of 200 over the Universe critical density) larger than $10^{15} M_{\odot}$, a medium-mass cluster $\left(M_{200}=3.510^{14} M_{\odot}\right)$ and three smaller systems, namely G676, G914 and G1542. Since these last three objects have very similar characteristics and behaviors, we chose to report our results only for the first one, as example. In Fig. 1 and Fig. 2 we show the projected maps for the spectroscopic-like temperature and for the metallicity, respectively. The map size is equal to the field of view of $X M M$-Newton, i.e. 30 arcmin: since we put our clusters at redshift $z=0.06$, this corresponds to $2088 \mathrm{kpc}$, using the cosmological model here assumed. The different metal maps refer to Iron and Oxygen (representative of the $\alpha^{-}$ elements). From these maps it appears that the largest clusters, G1.a and G51, have a rather complex structure, while the smaller cluster G676 appears as more relaxed, with G1.b representing an intermediate case.

In the following we briefly describe the dynamical state of our simulated clusters. This is fundamental to explain the sample characteristics which could influence the presence of any bias. The two massive clusters, G1.a and G51, both have an active dynamical state and strong inhomogeneities in the temperature maps (upper panels of Fig. (1). They show a monotonically increasing mass history, especially lately: this means that there is a significant amount of material which continuously in-falls inside the cluster virial region. All these small structures can be recognized both in the temperature maps (Fig. 11) and in the metallicity maps (Fig. 2) of these objects (see also the photon images reported in Fig. (3). Those are cold blobs which have not had enough time to thermalize in the hot ICM. Their presence does not affect the results of our analy- 
sis, since they have been identified and excluded from the $\mathrm{X}$-ray analysis (the masked regions are shown as green circles in Fig. 1, see Sect. 4). The metal maps show rather elongated structures, which trace the direction of the most recent merger events.

The cluster G1.b is close to G1.a, lying in the same resimulated Lagrangian region. It has experienced two consecutive important major merger events and it has not had enough time to relax. This clearly appears from the complex features in the temperature map (see also the presence on the photon image of a bright extended source on the West side of the centre). The core of the merged halo is detectable by eye from the metal maps. Quite interestingly, the Oxygen distribution can be used to reconstruct the merging dynamics.

The cluster G676 is an isolated system, which presented a relevant merging event at $z \approx 0.7$, but after then it has had time to relax. The object can be considered in dynamical equilibrium at present time, in the sense that its global velocity dispersion and its mass are almost constant in time after the merging event. Its homogeneous temperature map, round flux contours and spherical metallicity distribution confirm this picture. Similar considerations can be done for the other two small objects, G914 and G1542 (not shown in the figures).

\section{THE X-RAY ANALYSIS}

The simulated clusters have been processed through XMAS2 in order to produce mock observations with the EPIC-MOS1 and EPIC-MOS2 cameras and with an exposure time of $200 \mathrm{ksec}$. As mentioned before, the field of view is 30 arcmin, corresponding to $2088 \mathrm{kpc}$ in physical units. The images have been created by integrating along the line of sight a box of $10 \mathrm{Mpc}$ centered on the clusters. In our analysis we assume that $X M M$-Newton is perfectly calibrated.

\subsection{Spatial analysis}

We create the photon images in the $[0.7-2.0] \mathrm{keV}$ band and use them to detect the presence of compact X-ray bright gas regions. These corresponds to cool and very dense gas clumps present in the simulation and likely produced by overcooling effects. These clumps are generally not observed in real clusters so to avoid contamination we remove these regions from our analysis. For this purpose we used the wavelet decomposition algorithm proposed by Vikhlinin et al. (1998). Parameters are settled to localize sources with a peak above $4.5 \sigma$ and to identify the surrounding region above the $2.5 \sigma$ level. The selected areas are shown as green circles in the temperature maps (Fig. 1). In Fig. 3 we show the photons images, extracted in the $[0.7-2] \mathrm{keV}$ energy band, corrected by vignetting, out-of-time events, point spread function, background and out-field-of-view component, binned to 3.2 arcsec.

\subsection{Spectral analysis}

The spectral analysis has been performed by extracting the spectra from the event file in concentric annuli, centered on the brightest X-ray peak of the cluster. The first annulus has an internal radius of $50 \mathrm{kpc}$ (the central region is excluded from the analysis) and an external radius which is 1.25 times larger. All the remaining annuli are logarithmically equi-spaced with the same step of 1.25. During the spectrum extraction, we also subtracted all photons coming from the masked regions, already mentioned in the previous section, and from box regions containing the CCD gaps (clearly detectable as horizontal and vertical lines in the photon images displayed in Fig. 3). The spectra have been fitted using the $\mathrm{C}$ statistics in the XSPEC 11.3.2 package (Arnaud 1996) and following two procedures widely used by observers:

- Method 1. We fit the data with an absorbed VMEKAL model in the [0.4-8] keV energy band, leaving at the same time as free all the parameters of interest: temperature, Iron, Silicon, Magnesium, Oxygen and normalization. This approach is faster and more automatic but can lead to artificially biased results because of the degeneracy of the parameters.

- Method 2. This is a four steps procedure. (i) we fit the data with an absorbed MEKAL model in the $[0.4-8] \mathrm{keV}$ band to obtain the temperature; metallicity and normalization are considered free parameters; (ii) we fix the temperature and use a VMEKAL model in the same energy band to recover the Iron abundance; the other three metals are left free; (iii) we keep frozen temperature and Iron to measure the Oxygen in the $[0.4-1.5] \mathrm{keV}$ band and (iv) finally we freeze the values of temperature, Iron, Oxygen to estimate the Magnesium and Silicon abundances in the $\left[\begin{array}{ll}1.2 & 3.2\end{array}\right] \mathrm{keV}$ band.

In both fitting methods we fixed the Galactic absorption $N_{H}$ and the redshift to the input values used to simulate the observations with X-MAS2 (i.e. $N_{H}=5 \times 10^{20} \mathrm{~cm}^{-2}$ and $z=0.06)$.

\section{RESULTS}

From the spectral analysis we recovered the projected profiles for temperature, Iron, Silicon, Magnesium and Oxygen and we compared them to the values directly extracted from the hydrodynamical simulations. The simulation profiles are computed projecting the quantities along the line of sight, within the same radial bins used in X-ray analysis and masking out the same regions described in Sect.4. The temperature is computed using the spectroscopic-like definition, $T_{\mathrm{SL}}$ (Mazzotta et al. 2004), summing over all particles with temperature higher than $0.5 \mathrm{keV}$. For the metal profiles we use instead the emissionweighted formula:

$$
Z_{\mathrm{ew}}=\sum Z_{i} \times W_{i} / \sum W_{i}
$$

where $W_{i}$ is the emissivity of the $i$-th particle computed in the $[0.4-8] \mathrm{keV}$ energy band considering the contribution of each particle metals.

The results for G1.a, G51, G1.b, and G676 are presented in Fig. 4. The black lines refer to the simulated input values, while spectroscopic values determined from the full-band-fitting (Method 1) and the ones recovered in the narrow band are shown by the green and red lines, respectively. The vertical error bars are at $1 \sigma$ level while horizontal bars represent the width of the radial bin. 


\subsection{Temperature}

The recovered spectroscopic temperature matches well the input temperature leading to a maximum relative error of 5 per cent for hot systems. This difference is expected for objects with a complex temperature structure (see Fig. 1) and steep temperature gradients (see Fig. 4), as already delineated in Mazzotta et al. (2004). It is worth noting that the excellent agreement is also valid for the three cooler systems, for which the temperature falls from $2 \mathrm{keV}$ at $2 \operatorname{arcmin}(\sim 150 \mathrm{kpc})$ down to $1 \mathrm{keV}$ at 6 arcmin $(\sim 450 \mathrm{kpc})$. Indeed, this result is expected since these objects show a regular and homogenous temperature map and any weighting (i.e., mass-weighted, emission-weighted and spectroscopic-like) give the same consistent result in the limit of isothermal systems (Mazzotta et al. 2004).

As expected, we do not find any difference between the spectroscopic temperatures recovered using Methods 1 and 2 , i.e. between MEKAL and VMEKAL fitting. In fact we find that for the most massive clusters the two estimates almost perfectly coincide; in the other cases the differences are always smaller than 2 per cent.

\subsection{Iron}

Thanks to the powerful emission of both Fe-L and Fe-K lines, the spectroscopic determination of the Iron abundance is recovered with great accuracy and presents relatively small error bars. The strength of Fe-K lines for objects having a temperature larger than $3 \mathrm{keV}$ and the one of Fe-L lines for systems with temperature lower than $2 \mathrm{keV}$ (see Fig. 5) ensures an appreciable agreement for both cold and hot clusters. In each radial bin the Iron abundance derived spectroscopically matches the emission-weighted value from the original hydrodynamical simulation at $3 \sigma$ level. Moreover the relative difference, $\left[\mathrm{Fe}_{\text {spec }}-\mathrm{Fe}_{\text {sim }}\right] / \mathrm{Fe}_{\text {sim }}$, is less than 5 per cent. The only exception is for G1.b, the 2-3 keV cluster (see Fig. 4). For this object we detect a systematic overestimate with respect to the input profile which can be as high as 20 per cent. The reason of this discrepancy - related to the complex temperature structure of this cluster (already evident in the corresponding panel of Fig. (1) and to its particular temperature range - will be extensively discussed later in Sect. 6.2 Some spatial bins of G676 show an underestimate of Iron, which is between $10 \%$ and $30 \%$. This feature is caused by the Iron bias described in Buote (2000a). Among our cold clusters, G676 is the only one presenting this characteristic; we will further investigate this aspect in Sect. 6.3.

It is worth noting that the Iron spectroscopic abundances obtained from Method 1 and Method 2 are not significantly different. In fact, the two procedures differ just by keeping fixed the temperature in the second case, but the possible degeneracy between Iron and Temperature is weak because of the large number of counts we had (larger than $10^{4}$ ). The maximum discrepancy between the two methods is smaller than 10 per cent and it is present only for some radial bins of the coolest objects.

\subsection{Oxygen}

The spectroscopic measurements of Oxygen, as well as Magnesium and Silicon, are more uncertain in the inner regions of the clusters. The effect is larger for the hottest systems, G1.a and G51, and decreases for objects with smaller masses. The explanation resides on projection effects: in fact the spectrum of the central region is obtained by integrating along the whole line-of-sight, thus it includes contributions from plasma at temperatures which can significantly differ in the case of most massive clusters, because of their steeper radial profile. For the systems with temperature lower than $3 \mathrm{keV}$, the spectroscopic determination of the Oxygen abundance always agrees with the input values inside $1-2 \sigma$. On the other hand, for the largest clusters, G1.a and G51, the Oxygen detection is systematically overestimated, even if the large error bars reduce the statistical significance in most radial bins. Increasing the exposure time, i.e. the photon number, reduces the error and the systematic effect becomes more evident. To confirm this result, in Fig. 6] we report the spectroscopic result for G1.a, our hottest cluster, for which we simulated a 1 Msec exposure. From this plot we notice how the spectroscopic values significantly overestimate the input value by a factor of 3 .

As a general behavior, we notice that Oxygen obtained by fitting the spectra with Methods 1 and 2 are similar for the smallest objects and with discrepancies always inside the error bars. Nevertheless, there is a tendency of obtaining an abundances larger than the input one when Oxygen is measured using the broad band (Method 1 shown as green dashed points in Fig. (4), being the difference increasing with the temperature.

\subsection{Magnesium}

Generally speaking, the measurement of Magnesium is very difficult because, at the spectral resolution of $X M M$ Newton, its line energy is well within the energy range of the Fe-L line complex (see Sect. 5.6 and Fig. 5 ). In few cases it is really impossible to detect any Magnesium content, and when a detection is obtained we get always very large errors. Things improves slightly if we use Method 2 but not by much.

Magnesium lines are stronger for cool systems: in fact, in the cases of G1.b and G676 (see the third and fourth columns of Fig. (4), we are able to trace the overall profile, even if no strong constraint can be obtained. In any case for these cold clusters we do not find any systematic bias. On the contrary, the $6 \mathrm{keV}$ cluster, G51, shows large discrepancies in the external bins where the spectroscopic estimates are between 200 and 400 per cent higher than the input values (see Fig. 4). Finally, for G1.a no conclusion can be reached since its high temperature (all radial bins have values above $8 \mathrm{keV}$ ) makes the Magnesium extremely difficult to be detected (see also Fig. (5)).

\subsection{Silicon}

The difference between the results obtained using Methods 1 and 2 is significant also in the measurements of the Silicon abundances. Shrinking the energy band improves the estimates, mainly for temperature larger than $2-3 \mathrm{keV}$. For the cluster G1.b, the choice of a narrow energy band (red lines in Fig. (4) helps in preventing the underestimate of this metal by 40-50 per cent. G51 and G1.a show a similar tendency, even if this result is less significant because of the larger error bars. The colder clusters, instead, do not show any particular difference between the two spec- 
tral methods: in both cases the input abundance of Silicon is well recovered from the X-ray analysis at 1-1.5 $\sigma$ level.

In general the agreement between the X-ray results and the input values from the hydrodynamical simulations is remarkably good at all temperatures. It is important to say, however, that the silicon line energy is located in region where, due to a combination of CCD and mirrors, the effective area falls down rapidly and after $2 \mathrm{keV}$ it shows a strong edge due to Au and Ir. For real observations this may be a problem as this spectral region of the detector is very difficult to calibrate. However, this problem will not be present for high-redshift clusters, for which the Silicon line is redshifted towards the softer region.

\subsection{Thermal Breemstrahlung spectra as seen by XMM-Newton}

Our main goal is to investigate how well we can measure the metal abundances with the availability of the XMMNewton EPIC camera spectra. In order to better understand the results of our analysis we illustrate here some general features of thermal breemstrahlung spectra observed with the same spectral resolution of XMM-Newton detectors. We generate with XSPEC six spectra at different values of temperature: $1,2,3,5,8$, and $10 \mathrm{keV}$. These are convolved with the instrumental response of the MOS-1 camera (almost identical results can be obtained considering MOS-2). The adopted plasma model is VMEKAL, for which we set to zero the abundances of all metals, except $\mathrm{O}, \mathrm{Mg}, \mathrm{Si}$ and $\mathrm{Fe}$, which are fixed to their solar values. The resulting spectra are shown as red lines in Fig.5. In the same plot different color lines refer to the separate contribution of each metal: Oxygen (green), Magnesium (blue), Silicon (cyan) and Iron (black). Decreasing the plasma temperature, we can notice that the emission lines become more evident and the ratio between Fe-L (Iron lines around $1 \mathrm{keV}$ ), and $\mathrm{Fe}-\mathrm{K}$ (Iron lines around $6 \mathrm{keV}$ ), rapidly increases. The Oxygen lines, due to both OVII and OVIII lines, show two bumps around $0.6-0.8 \mathrm{keV}$ which are more evident for systems with temperature lower than $3 \mathrm{keV}$. The Oxygen lines are located very close to the Fe-L complex, whose tail in the soft energy region, given the spectral resolution of $X M M-N e w t o n$, can interfere with Oxygen determination for systems with temperature around 1-2 keV. At temperatures larger than $3 \mathrm{keV}$, the Oxygen and Fe$\mathrm{L}$ features are well separated. The Magnesium lines are quite strong only for very cold systems with temperature around 1-3 keV. Nevertheless, also in this case, the spectral resolution of the XMM-Newton EPIC-MOS cameras is not good enough to allow to distinguish this line from the Fe-L complex, which has an extremely powerful emission in this temperature range. The importance of the Magnesium lines is rapidly decreasing. Silicon, instead is present in a more isolated region of the spectrum (close to $2 \mathrm{keV}$ ), therefore this guarantees a good measurement of its abundance for systems with temperature up to approximately $8 \mathrm{keV}$. Another important property of the energy region around $2 \mathrm{keV}$ is that different temperature spectra with the same normalization have there the same flux. This means that the Silicon measurement is not significantly influenced by the presence of a multi-temperature plasma or, in other words, by the uncertainties related to the continuum determination.
It is important to stress that the possibility of accurately determining the emission lines is subject not only to the mutual interaction of different elements, but also to the capability of measuring with accuracy the Iron lines (which can strongly influence Oxygen and Magnesium) and the continuum.

\section{DISCUSSION}

\subsection{Differences between the methods used in the spectral analysis}

Considering the results presented in Sect. 5] (see also Fig. 4), we can conclude that the two strategies followed in the X-ray analysis give almost identical estimates in the case of temperature and Iron. The only relevant difference is that in Method 2 we freeze the temperature to compute the Iron. The effect is to eliminate one free parameter, reducing the degeneracy, which, however, is extremely weak in our case, because of the large number of counts per each radial bin. The most evident differences between the two procedures are for the abundance measurements of the $\alpha$-metals (Oxygen, Silicon and Magnesium): in all cases we obtain a better result when we fix the continuum and the Iron contribution and we fit the spectrum in a narrow band centered on the corresponding set of lines. We made several tests to individuate the optimal energy band to adopt. At the assumed redshift, i.e. $z=0.06$, the best choices are [0.4-1.5] keV for Oxygen and [1.2-3.2] keV for both Magnesium and Silicon. Notice that the bands need to be large enough to include part of the continuum to better estimate the line emission and to evaluate the effects of each single element on the continuum. In particular this shrewdness is very important for Oxygen, for which the continuum contribution is relevant on a wide energy range (see the green line in the first panel of Fig. 51). Using Method 2 instead of Method 1 allows to avoid an overestimated result for Oxygen. Moreover it permits to detect Magnesium which would not be recovered without freezing the Iron. Finally it increases the spectroscopic determination of Silicon avoiding a possible underestimate.

\subsection{Iron overestimate for systems at 2-3 keV}

The Iron content of the ICM is perfectly recovered for hot systems, G1.a and G51, because their Fe-K lines are extremely well determined (see Fig. 4 and the last 3 panels of Fig. 5). Iron is also accurately estimated for cold systems (only exception are some bins of G676 in Fig. 4- see Sect. 6.3), since their Fe-L lines are extremely strong (see the first two panels of Fig. [5). The only system for which we obtain a systematic overestimate in all radial bins is G1.b, the 2-3 keV object. Notice that this discrepancy is significant, as evident from the error bars in the corresponding panel of Fig. 4. The problem is due to the fact that the G1.b temperature is very close to the one where there is a transition between the relative importance of the lines (Fe-L or Fe-K) used in the determination of the global Iron content. Moreover, its temperature structure is quite complex: consequently inside the same radial bin we are averaging different temperatures which sometimes are characterized by a large Fe-K contribution, while other times show a strong Fe-L. Summing over spectra with both high Fe-L and high Fe-K has the net effect of increasing the final amount of Iron. This effect can be explained by 
Rasia et al.

looking at Fig. 7, In the first panel we combine together a plasma at $1 \mathrm{keV}$ (green line) with a plasma at $4 \mathrm{keV}$ (dark line): the resulting spectrum is shown by the red line. It is evident that the first plasma contributes to bump the Fe-L lines, while the second one has the role of bursting the Fe-K lines in the resulting global spectrum, which is then characterized by an Iron content which is larger than what expected considering its temperature.

In order to quantify this effect we perform a test with a likely composition of the gas (see the lower panel of the same figure). The input data is the spectrum resulting from the combination of two plasmas: the first one with temperature $T_{1}=2 \mathrm{keV}$, metallicity $Z_{1}=0.2$ and normalization $K_{1}=1$; the second one with $T_{2}=3 \mathrm{keV}, Z_{2}=0.1$ and $K_{2}=1$. We produce few spectra with different number counts and fit them with a $\mathrm{C}$ statistics. The results are reported in Table 3. They show a perfect consistency for the temperature, but a significant overestimate of the Iron spectral value with respect to the emission-weighted one. The discrepancy is highly significant and depends on the number counts: for 4.500 counts the disagreement is of almost $40 \%$. Increasing the statistic number the difference is alleviated but not solved. Even observing the cluster for $2 \times 10^{6}$ sec and thus having $9 \times 10^{5}$ counts (which is 1 2 orders of magnitude above the typical observed counts rate, see e.g. Balestra et al. 2007), the discrepancy is still present and of order of $15 \%$. The spectrum corresponding to the best-fit (red line) of the $4.5 \times 10^{5}$ counts spectrum is compared to the input data in the same panel of Fig:7. while in the bottom box we show the corresponding residuals. Notice that in the residual behavior there are not evident features which can make us suspicious that the fit is not good or that it can be affected by some bias on the Iron lines.

The implications of these results are extremely important. Iron is in fact the metal to which all the other $\alpha^{-}$ elements are referring, thus overestimating its content can lead to wrong conclusions on the past stellar population.

This effect may also explain, at least partially, the recent results obtained by Baumgartner et al. (2005). After stacking in temperature bins 273 clusters observed with $A S C A$, they studied the global metal abundances as a function of the mean temperature. They found that in all temperature bins (from $0.5 \mathrm{keV}$ to $12 \mathrm{keV}$ ) the emissionweighted Iron abundance within the cluster region selected to contain as much flux as possible is around 0.2-0.25 (in solar units), with the only exception of the two bins between 2 and $4 \mathrm{keV}$, where a global Iron value of $0.43-0.47$ $Z_{\odot}$ is measured.

\subsection{Fe bias for cold systems}

Another bias affecting the estimate of the Iron content is present in some bins of the cold system G676 (see Fig. (4). The systematic underestimate is know in literature as "Iron bias" (Buote 2000a,b). However, this bias gives at maximum an underestimate of order of $20 \%$ and it is present only in G676, but it does not influence the other two cold systems. The poor evidence of this bias in our sample can be explained as follow.

The Iron bias introduced by Buote (2000a) is caused by the fact of pretending to fit with a single temperature model a plasma which is, instead, characterized either by a combination of different temperatures (multi-temperature plasma) or by a strong temperature gradient. Observationally, the bias is seen to affect the central cooling core regions where typically there is a positive temperature gradient $(d T / d r>0)$ as well as both negative metallicity gradient $(d Z / d r<0)$ and negative emission gradient $(d E M / d r<0)$. The combination of these factors leads to enlarge the bump of Fe-L shell (the colder plasma with higher metallicity and emission excites the lines on the soft part of Fe-L shell). In our work, we are not in this regime. On the contrary, both temperature and metallicity gradient are shallow inside the spatial bins we considered. Moreover the temperature gradient in the our external regions is going in the opposite direction $(d T / d r<0)$ with respect its behavior in the cooling regions.

Another reason for which we do not detect a strong $\mathrm{Fe}$ bias in our system is because it decreases if the spectral fitting is made in an energy band sufficiently large to determine the continuum or, in other words, the temperature profile (Buote 2000b) with accuracy. Buote et al. (2003) suggested to extend the minimum of the fitting energy band down below $0.6 \mathrm{keV}$ in order to limit the effect of this bias. With our analysis we respect this demand since the minimum energy considered is $0.4 \mathrm{keV}$. In addition, a robust determination of the continuum is guaranteed by the high signal to noise that we have up to $4 \mathrm{keV}$. The fact that a good Iron measurement depends on a correct temperature measurement is clear comparing in Fig. 4 the G676 temperature and Iron panels.

\subsection{Oxygen and Magnesium for hot clusters}

In Sect. 5 we showed that the abundance estimates for both Oxygen and Magnesium do not show any problem of systematics for systems colder than $2 \mathrm{keV}$. On the other hand, for the hot clusters, like G1.a and G51, we found a clear bias producing a significant overestimate. Notice that these elements are the best $\alpha$-element indicators, thus they are a fundamental tool for studying the stellar evolution and formation history through the ratio between $\mathrm{SNe}$ Ia and SNe II. It is therefore necessary to understand in detail the origin of the discrepancy. At this aim we investigate a number of possible reasons which depend on the ICM physics implemented in the original hydrodynamic simulation, on the complexity of the plasma temperature and on the features of the X-ray spectra.

- Presence of small undetected cold blobs. Clusters G1.a and G51 show in their photon images (Fig. 3) a large number of compact gas clouds spread in the ICM which are usually associated to cold blobs in the temperature maps (Fig. 1). Most of them have been detected by the wavelet decomposition algorithm and then excluded from our analysis, but some of them are still present, in particular in the external regions. These structures, which have a very low temperature, could in principle be responsible for an overestimate for Oxygen or Magnesium, since their importance is greater for cold systems. To investigate this problem in more detail we generate new event files for G1.a and G51, but using only the particles having a temperature larger of $5 \mathrm{keV}$ : in this way we are sure to avoid any 
contamination from cold (diffuse and clumped) gas. To these event files we apply the whole procedure describe above. As expected, using the wavelength algorithm, we do not find any compact cool source and we just exclude the second brightest blob, located on the East side of G51 centre. The comparison between the spectroscopic results and the simulation profiles of Oxygen for G1.a and Magnesium for G51 (re-computed excluding the particles with temperature smaller than $5 \mathrm{keV}$ ) are shown in Fig. 8. it is clear that the discrepancies are not alleviated.

- Dynamical state of the cluster. Since the bias is evident only for the two largest clusters which clearly have a complex morphological structure, while it does not affect the coolest systems which are more symmetric and regular, its relevance could be enhanced by the presence of thermal inhomogeinities in the ICM. However, we notice that G1.b does not show this phenomenon, even if it represents a perturbed object. To further investigate this possibility, we generate another series of events files fixing the temperature of all the particles at a given constant value $(8 \mathrm{keV})$ but letting all the metals equal to their original values. In this way we create an object with a completely flat temperature structure. We repeat the analysis on these new images, finding again the same discrepancy for the Oxygen and Magnesium abundances.

- Fe-L over Fe-K ratio. The spectra we have extracted from the images are the sum of contributions coming from the plasma present along the line of sight and having different temperatures and metallicities. The resulting spectra, therefore, could have a Fe-L over Fe-K ratio different from the typical value reported by the VMEKAL model in correspondence of the measured temperature. The Iron content of hot systems is estimated basically using the Fe-K systems which are strongly more powerful than the Fe-L lines at those temperatures. The Fe-L group of the spectra can be, thus, weaker than what we expect from a VMEKAL model where we estimate the Iron content by fitting the Fe-K lines. In this situation one way to "fill the gap" between the true Fe-L of the spectra and the expected one of the model is to increase the emission power by other elements present in the same energy band. We performed a detailed comparison of the spectral Fe-L and Fe-K measurements and the corresponding model expectations. We conclude that the Magnesium estimate can be affected for this reason. In fact for G51, which is a system showing a large disagreement for $\mathrm{Mg}$ lines, the Iron determination from the Fe-L is always higher than what we derive from the Fe-K lines. On the other hand, this behavior is not present in G1.a and thus cannot explain the disagreement found for Oxygen, which is also displaced by Fe-L at temperatures larger than $8 \mathrm{keV}$ (see Fig. 5).

\section{- Continuum determination.}

Oxygen is a weak line at temperatures larger than 5 $\mathrm{keV}$, thus a wrong determination of the continuum can strongly influence the measurement of this element. In particular an underestimate of the continuum can originate an overestimate of the Oxygen abundance. To have a rough estimate of this effect we simulate a spectrum as sum of a $6 \mathrm{keV}$ plasma with Oxygen equal to 0.1 (in solar units) and a $8 \mathrm{keV}$ plasma with Oxygen fixed to 0.2. The two plasmas have been weighted in the same way by assigning a unity normalization to both. The fake spectrum has been convolved with the $X M M$ Newton response and simulated assuming an exposure time of $1 \mathrm{Msec}$. This large integration time allows us to have few millions of counts per spectra. We performed a first fit using a $\chi^{2}$ statistics and a VMEKAL model where we let free temperature, Oxygen and normalization: the resulting temperature agrees with the spectroscopic-like estimate. This temperature has been then fixed in the following tests, where we also fixed the normalization, but to different values, starting the input theoretical value of 2 and then decreasing it slightly every time. In this way we are able to study the effect that only a wrong determination of the continuum has on the spectroscopic determination of Oxygen. The expected emission-weighted abundance for Oxygen is 0.149 . This value is perfectly recovered when we force the normalization to be the theoretical one. A result compatible at $1 \sigma$ level is still obtained using a lower normalization, up to 1.995. Decreasing further the normalization by 0.5 or 0.75 per cent, the spectroscopic determination of Oxygen becomes much larger than the expected one. This simple test shows us how Oxygen is very dependent on the goodness of the continuum determination and suggests that the measurements done through XMMNewton in high-temperature systems can be significantly biased.

\section{CONCLUSIONS}

The Intracluster Medium represents a perfect laboratory to study the physics of all the cluster subcomponents, like its galaxies and the stars in galaxies. In particular the ICM metallicity is a rich source of information to test the stellar evolution models. In this work we deeply investigated all possible systematic biases present in the metallicity measurements from X-ray spectra having the same spectral resolution of XMM-Newton. At this aim we improved the X-ray MAp Simulator in order to correctly consider the metal information which is provided by our simulations. The six clusters here analyzed cover a wide range of temperature and have different dynamical state. This allows us to determine how the biases we analyzed are effectively influenced by the complexity of the thermal structure and by the value of the temperature itself.

Performing the X-ray analysis we tested two standard procedures adopted by X-ray observers: first, we fitted the spectra in a large band leaving free at the same time all the parameters of interest ( $T, \mathrm{Fe}, \mathrm{O}, \mathrm{Mg}, \mathrm{Si})$; second, we recover the temperature and the Iron content from a fit in a large band and then we measure the $\alpha$-elements, O, 
$\mathrm{Mg}$, and $\mathrm{Si}$, in a narrower band centered on the lines. Our main results are shown in Fig. 4 and can be summarized as follows:

- Using narrow bands to measure all the elements beside Iron is in general better for all clusters, independently of their temperature. This procedure prevents to overestimate Oxygen and to underestimate Silicon. Furthermore it increases the probability to detect the Magnesium content.

- Iron is perfectly recovered as soon as either Fe-K or Fe-L are the dominating lines: this occurs when the plasma has a temperature larger than $3 \mathrm{keV}$ or smaller than $2 \mathrm{keV}$, respectively. In our sample we have an object showing a complex map temperature (Fig. 1) with values ranging between 2-3 keV (see its temperature profile in Fig. (4). Simulating a simple model which describes this system in XSPEC we found that the spectroscopic Iron is overestimated by up to 15-40 per cent depending on the number statistic: decreasing the source counts leads to an increase of the disagreement. This result has strong implications. In fact one of the main goals of measuring the ICM metallicity is the derivation of the $[\alpha / \mathrm{Fe}]$ and $[\mathrm{Si} / \mathrm{Fe}]$ ratios (see the Introduction). Therefore, the fact that we detect a clear systematic effect in the Iron measurements can affect the results that we can derive from them about the stellar formation history.

- Oxygen is well measured for clusters with temperature lower than $3 \mathrm{keV}$, while we find an evidence of overestimate for the hottest system in our sample, which has a temperature profile ranging from 8 to $12 \mathrm{keV}$ (see Fig. 4). We check carefully some of the possible reasons that can originate this effect. The explanation came out to be the problematic balancing between the determination of the continuum and the weakness of this line at temperature higher than $8 \mathrm{keV}$. A discrepancy smaller than 1 per cent on the normalization is sufficient to explain the Oxygen overestimate.

- The measurement of Magnesium is difficult at all temperatures: for cold objects (with $T<2 \mathrm{keV}$ ) its line is, in fact, lying in the same spectral range of strong Fe-L lines and thus its detection is hidden, while at high temperatures it is a very weak line. We found a systematic overestimate of Magnesium in hot clusters: this effect can be related to the fact that the $[\mathrm{Fe}-\mathrm{L} / \mathrm{Fe}-\mathrm{K}]$ ratio is higher in the spectra than in the model used to fit them. Thus to fill the Fe-L group lines there is the necessity to pump the emission of other elements present in the same energy band, that in our case, is Magnesium.

- Finally, from the X-ray analysis of the spectra we can measure with good precision Silicon for all the objects in our sample independently of their dynamical state. This is due to the fact that the value of the spectra around $2 \mathrm{keV}$ is only slightly dependent on the temperature.

The method used in this paper to investigate the metallicity distribution takes an enormous advantage by the use of high-resolution simulations where the hydrodynamic physics is treated including many different physical processes which are relevant for the thermal and chemical evolution of the ICM. Other aspects which can play a significant role, like AGNs, cosmic rays and magnetic field, are not included in these simulations but could be added in the next future. At the present the state of our simulations does not fully reproduce the cluster core, where more of the X-ray information comes from, but it is a proper description of the temperature gradient outside 0.1 $R_{200}$ (Pratt et al. 2007). Nevertheless, it is worth stressing that our results and the corresponding explanations are not dependent on the kind of simulations used. In fact, when we found some disagreement between the input profiles from our simulation and the X-ray spectroscopic measurements, we investigated the problem by analyzing the spectral properties of fake plasma spectra generated using XSPEC . For this reason our results can be considered valid for observations of spectra generated by a plasma with a multi-component temperature. The only restrictions of our results are due to the fact that we focused our effort on a fixed spectral energy resolution, like the XMM-Newton one. The powerful of the analysis of the ICM metal content can radically change in the next future with an X-ray satellite having a micro-calorimeter on board. EDGE (Explorer of Diffuse Emission and Gammaray burst Explosions) 14 and Constellation-X $X^{15}$ are two projects that have been proposed with the intent of incorporating such technology, that will allow to solve the blending of Fe-L and Oxygen lines.

\section{ACKNOWLEDGMENTS}

We thank the referee for useful comments.

The simulations have been performed with CPU time allocated at the "Centro Interuniversitario del Nord-Est per il Calcolo Elettronico" (CINECA, Bologna) thanks to grants from INAF and from the University of Trieste. Support for this work was provided by the INFN grant PD-51 and by NASA through Chandra Postdoctoral Fellowship grant number PF6-70042 awared by the Chandra X-ray Center, which is operated by the Smithsonian Astrophysical Observatory for NASA under contract NAS803060. We acknowledge the financial support from contract ASI-INAF I/023/05/0. ER thanks for the hospitality the MPA in Garching, where part of the analysis was done. PM acknowledge support from NASA grants GO4-5155X and GO5-6124X. We are extremely grateful to Jean-Luc Sauvageot for sharing with us a previous version of a code which produced XMM-Newton images; to Mauro Roncarelli, Eugenio Ursino and Massimiliano Galeazzi for discussing on the new distribution technique; to Alexey Vikhlinin for providing information on the wavelet detection algorithm; to Kyoko Matsushita and Fabio Gastaldello for kindly discussions on X-ray metallicity analysis.

${ }^{14}$ http://projects.iasf-roma.inaf.it/edge/

15 http://constellation.gsfc.nasa.gov/index.html 
REFERENCES

Anders, E. \& Grevesse, N. 1989, Geochimica et Cosmochimica Acta, 53,197

Arnaud, K. A. 1996, in ASP Conf. Ser. 101: Astronomical Data Analysis Software and Systems V, 17-+

Balestra, I., Tozzi, P., Ettori, S., Rosati, P., Borgani, S., Mainieri, V., Norman, C., \& Viola, M. 2007, A\&A, 462, 429

Baumgartner, W. H., Loewenstein, M., Horner, D. J., \& Mushotzky, R. F. 2005, ApJ, 620, 680

Bertone, S., De Lucia, G., \& Thomas, P. A. 2007, preprint, astro-ph/0701407

Bohringer, H., Matsushita, K., Churazov, E., Finoguenov, A., \& Ikebe, Y. 2004, A\&A, 416, L21

Böhringer, H., Matsushita, K., Churazov, E., Ikebe, Y., \& Chen, Y. 2002, A\&A, 382, 804

Böhringer, H., Matsushita, K., Finoguenov, A., Xue, Y., \& Churazov, E. 2005, Advances in Space Research, 36, 677

Bourdin, H., Sauvageot, J.-L., Slezak, E., Bijaoui, A., \& Teyssier, R. 2004, A\&A, 414, 429

Buote, D. A. 2000a, MNRAS, 311, 176

Buote, D. A. 2000b, ApJ, 539, 172

Buote, D. A., Lewis, A. D., Brighenti, F., \& Mathews, W. G. 2003, ApJ, 595, 151

Cora, S. A. 2006, MNRAS, 368, 1540

De Grandi, S., Ettori, S., Longhetti, M., \& Molendi, S. 2004, A\&A, 419,7

De Grandi, S. \& Molendi, S. 2001, ApJ, 551, 153

De Lucia, G., Kauffmann, G., \& White, S. D. M. 2004, MNRAS, 349, 1101

de Plaa, J., Werner, N., Bleeker, J. A. M., Vink, J., Kaastra, J. S., \& Méndez, M. 2007, A\&A, 465, 345

de Plaa, J., Werner, N., Bykov, A. M., Kaastra, J. S., Méndez, M., Vink, J., Bleeker, J. A. M., Bonamente, M., \& Peterson, J. R. 2006, A\&A, 452, 397

Dolag, K., Vazza, F., Brunetti, G., \& Tormen, G. 2005, MNRAS, 994

Domainko, W., Mair, M., Kapferer, W., van Kampen, E., Kronberger, T., Schindler, S., Kimeswenger, S., Ruffert, M., \& Mangete, O. E. 2006, A\&A, 452, 795

Dupke, R. \& White, III, R. E. 2003, ApJL, 583, L13

Ettori, S., Fabian, A. C., Allen, S. W., \& Johnstone, R. M. 2002, MNRAS, 331, 635

Finoguenov, A., Arnaud, M., \& David, L. P. 2001, ApJ, 555, 191

Finoguenov, A., David, L. P., \& Ponman, T. J. 2000, ApJ, 544, 188

Finoguenov, A. \& Jones, C. 2000, ApJ, 539, 603

Finoguenov, A., Jones, C., Forman, W., \& David, L. 1999, ApJ, 514, 844

Finoguenov, A., Matsushita, K., Böhringer, H., Ikebe, Y., \& Arnaud, M. 2002, A\&A, 381, 21

Finoguenov, A. \& Ponman, T. J. 1999, MNRAS, 305, 325

Fukazawa, Y., Makishima, K., Tamura, T., Ezawa, H., Xu, H., Ikebe, Y., Kikuchi, K., \& Ohashi, T. 1998, PASJ, 50, 187

Fukazawa, Y., Makishima, K., Tamura, T., Nakazawa, K., Ezawa, H., Ikebe, Y., Kikuchi, K., \& Ohashi, T. 2000, MNRAS, 313, 21

Fukazawa, Y., Ohashi, T., Fabian, A. C., Canizares, C. R., Ikebe, Y., Makishima, K., Mushotzky, R. F., \& Yamashita, K. 1994, PASJ, 46, L55

Gardini, A., Rasia, E., Mazzotta, P., Tormen, G. and De Grandi, S., \& Moscardini, L. 2004, MNRAS, 351, 505

Gastaldello, F. \& Molendi, S. 2002, ApJ, 572, 160

Haardt, F. \& Madau, P. 1996, ApJ, 461, 20

Humphrey, P. J., Buote, D. A., Gastaldello, F., Zappacosta, L., Bullock, J. S., Brighenti, F., \& Mathews, W. G. 2006, ApJ, 646, 899

Lia, C., Portinari, L., \& Carraro, G. 2002, MNRAS, 330, 821

Liedahl, D. A., Osterheld, A. L., \& Goldstein, W. H. 1995, ApJL, 438, L115

Matsushita, K., Böhringer, H., Takahashi, I., \& Ikebe, Y. 2007a, A\&A, 462, 953

Matsushita, K., Finoguenov, A., \& Böhringer, H. 2003, A\&A, 401,

Matsushita, K., Fukazawa, Y., Hughes, J. P., Kitaguchi, T., Makishima, K., Nakazawa, K., Ohashi, T., Ota, N., Tamura, T., Tozuka, M., Tsuru, T. G., Urata, Y., \& Yamasaki, N. Y. 2007b, PASJ, 59, 327
Matsushita, K., Ohashi, T., \& Makishima, K. 2000, PASJ, 52, 685

Maughan, B. J., Jones, C., Forman, W., \& Van Speybroeck, L. 2007, ArXiv Astrophysics e-prints, astro-ph/0703156

Mazzotta, P., Rasia, E., Moscardinı, L., \& Tormen, G. 2004, MNRAS, 354, 10

Mewe, R., Gronenschild, E. H. B. M., \& van den Oord, G. H. J. 1985, A\&A Supp., 62, 197

Mewe, R., Lemen, J. R., \& van den Oord, G. H. J. 1986, A\&A Supp., 65,511

Molendi, S. \& Gastaldello, F. 2001, A\&A, 375, L14

Moll, R., Schindler, S., Domainko, W., Kapferer, W., Mair, M., van Kampen, E., Kronberger, T., Kimeswenger, S., \& Ruffert, M. 2007, A\&A, 463, 513

Monaghan, J. J. \& Lattanzio, J. C. 1985, A\&A, 149, 135

Morrison, R. \& McCammon, D. 1983, ApJ, 270, 119

Mushotzky, R., Loewenstein, M., Arnaud, K. A., Tamura, T., Fukazawa, Y., Matsushita, K., Kikuchi, K., \& Hatsukade, I. 1996, ApJ, 466, 686

Nagashima, M., Lacey, C. G., Baugh, C. M., Frenk, C. S., \& Cole, S. 2005, MNRAS, 358, 1247

Pratt, G. W., Böhringer, H., Croston, J. H., Arnaud, M., Borgani, S., Finoguenov, A., Temple, R. F. 2007, A\&A, 461, 71

Rasia, E., Mazzotta, P., Borgani, S., Moscardini, L., Dolag, K., Tormen, G., Diaferio, A., \& Murante, G. 2005, ApJL, 618,L1

Rasia, E., Ettori, S., Moscardini, L., Mazzotta, P., Borgani, S., Dolag, K., Tormen, G., Cheng, L. M., \& Diaferio, A. 2006, MNRAS, 369, 2013

Renzini, A., Ciotti, L., D'Ercole, A., \& Pellegrini, S. 1993, ApJ, 419, 52

Romeo, A. D., Sommer-Larsen, J., Portinari, L., \& AntonuccioDelogu, V. 2006, MNRAS, 371, 548

Sakelliou, I., Peterson, J. R., Tamura, T., Paerels, F. B. S., Kaastra, J. S., Belsole, E., Böhringer, H., Branduardi-Raymont, G., Ferrigno, C., den Herder, J. W., Kennea, J., Mushotzky, R. F., Vestrand, W. T., \& Worrall, D. M. 2002, A\&A, 391, 903

Salpeter, E. E. 1955, ApJ, 121, 161

Sanders, J. S. \& Fabian, A. C. 2006, MNRAS, 371, 1483

Sanders, J. S., Fabian, A. C., Allen, S. W., \& Schmidt, R. W. 2004, MNRAS, 349, 952

Saro, A., Borgani, S., Tornatore, L., Dolag, K., Murante, G., Biviano, A., Calura, F., \& Charlot, S. 2006, MNRAS, 373, 397

Sato, K., Yamasaki, N. Y., Ishida, M., Ishisaki, Y., Ohashi, T., Kawahara, H., Kitaguchi, T., Kawaharada, M., Kokubun, M., Makishima, K., Ota, N., Nakazawa, K., Tamura, T., Matsushita, K., Kawano, N., Fukazawa, Y., \& Hughes, J. P. 2007, preprint, astro-ph/0701328

Schindler, S., Kapterer, W., Domainko, W., Mair, M., van Kampen, E., Kronberger, T., Kimeswenger, S., Ruffert, M., Mangete, O., \& Breitschwerdt, D. 2005, A\&A, 435, L25

Smith, R. K., Brickhouse, N. S., Liedahl, D. A., \& Raymond, J. C. 2001, ApJL, 556, L91

Springel, V. 2005, MNRAS, 364, 1105

Springel, V. \& Hernquist, L. 2002, MNRAS, 333, 649

-. 2003, MNRAS, 339, 289

Sutherland, R. S. \& Dopita, M. A. 1993, ApJS, 88, 253

Tamura, T., Bleeker, J. A. M., Kaastra, J. S., Ferrigno, C., \& Molendi, S. 2001, A\&A, 379, 107

Tamura, T., Kaastra, J. S., den Herder, J. W. A., Bleeker, J. A. M., \& Peterson, J. R. 2004, A\&A, 420, 135

Tormen, G., Bouchet, F. R., \& White, S. D. M. 1997, MNRAS, 286 , 865

Tornatore, L., Borgani, S., Dolag, K., \& Matteucci, F. 2007, MNRAS

Tornatore, L., Borgani, S., Matteucci, F., Recchi, S., \& Tozzi, P. 2004, MNRAS, 349, L19

Valdarnini, R. 2003, MNRAS, 339, 1117

Vikhlinin, A. 2006, ApJ, 640, 710

Vikhlinin, A., McNamara, B. R., Forman, W., Jones, C., Quintana, H., \& Hornstrup, A. 1998, ApJ, 502, 558

Werner, N., Böhringer, H., Kaastra, J. S., de Plaa, J., Simionescu, A., \& Vink, J. 2006, A\&A, 459, 353

Yoshida, N., Sheth, R. K., \& Diaferio, A. 2001, MNRAS, 328, 669 

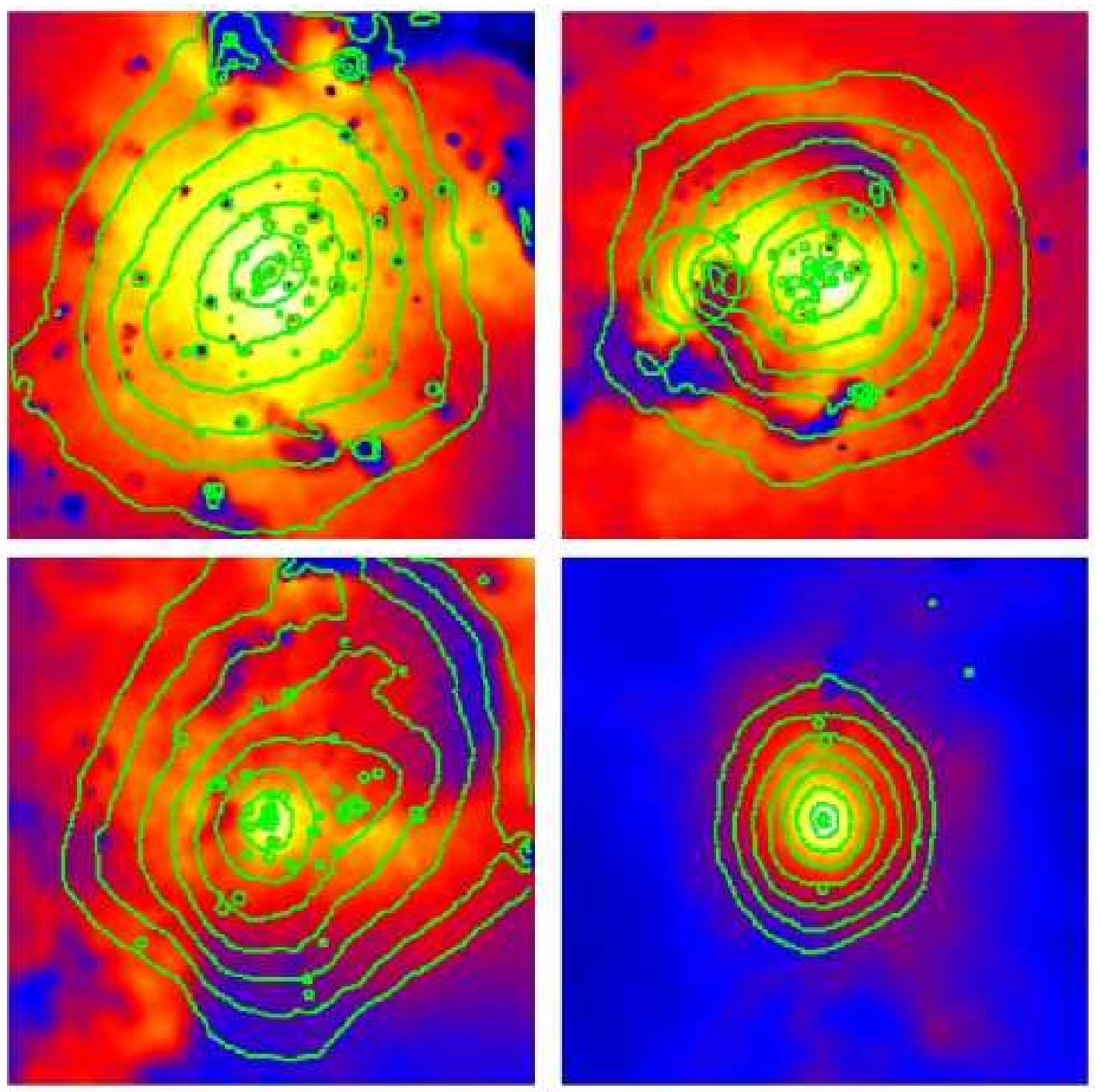

FIG. 1.- Spectroscopic-like temperature maps for the mock clusters: G1.a (top-right panel), G51 (top-left panel), G1.b (bottom-right panel), G676 (bottom-left panel). The color scale is linear, ranging from a minimum value of $0.1 \mathrm{keV}$ up to a maximum value, which is equal to $13,11,4.5,3 \mathrm{keV}$ for G1.a, G51, G1.b and G676, respectively. We overplot (black curves) the flux contours (level equi-spaced by a factor of 2) and the compact X-ray bright cool regions detected using the wavelet decomposition algorithm (green circles).

TABLE 1

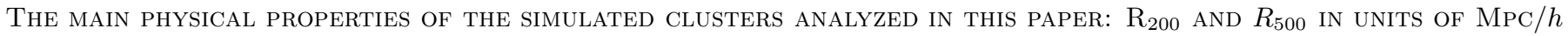
(THE RADIUS OF THE SPHERES WITH A DENSITY 200 AND 500, RESPECTIVELY, TIMES THE CRITICAL DENSITY OF THE UNIVERSE); THE MASSES INSIDE THESE RADII, $M_{200}$ AND $M_{500}$, IN UNITS OF $10^{14} M_{\odot} / h$, AND THE MASS-WEIGHTED

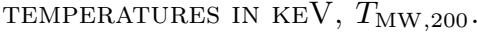

\begin{tabular}{lccccc} 
& $R_{200}$ & $R_{500}$ & $M_{200}$ & $M_{500}$ & $T_{\mathrm{MW}, 200}$ \\
\hline G1.a & 1.771 & 1.202 & 12.92 & 10.09 & 7.37 \\
G51 & 1.675 & 1.120 & 10.92 & 8.16 & 6.44 \\
G1.b & 1.151 & 0.740 & 3.55 & 2.36 & 2.63 \\
G676 & 0.727 & 0.490 & 0.89 & 0.68 & 1.42 \\
G914 & 0.718 & 0.485 & 0.86 & 0.66 & 1.39 \\
G1542 & 0.725 & 0.484 & 0.88 & 0.66 & 1.32
\end{tabular}



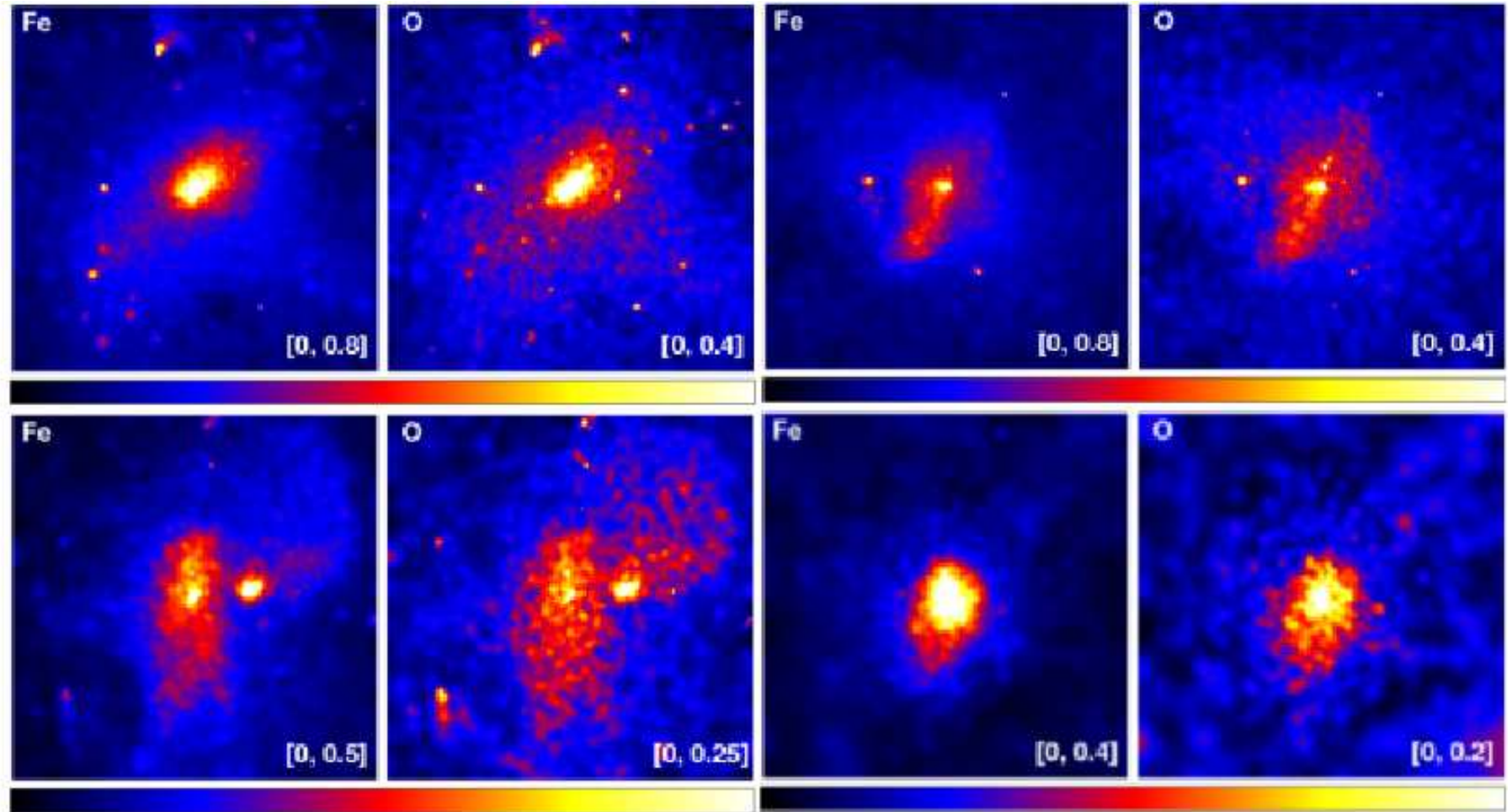

FIG. 2.- Iron and Oxygen distribution map for the same clusters shown in Fig. 1. G1.a (top-right panel), G51 (top-left panel), G1.b (bottom-right panel), G676 (bottom-left panel). The linear color scale (in solar units, Anders \& Grevesse) is reported in each panel.

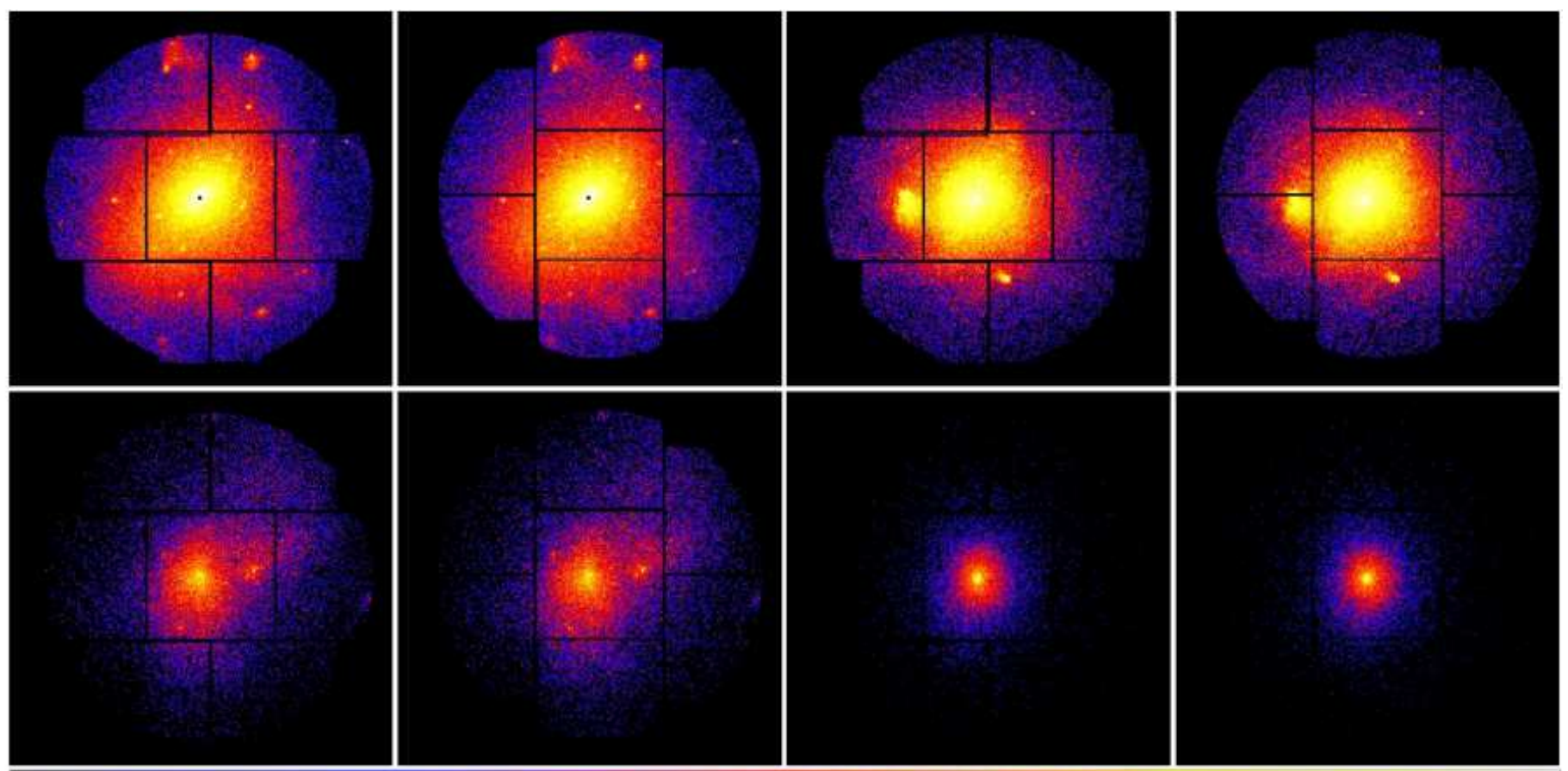

FIG. 3.- Photons images in the [0.7-2] keV energy band of the clusters in fig 1 G1.a (top-right panel), G51 (top-left panel), G1.b (bottom-right panel), G676 (bottom-left penal). The images are binned to 3.2 arcsec, background subtracted, and corrected for vignetting and out-of-time events 


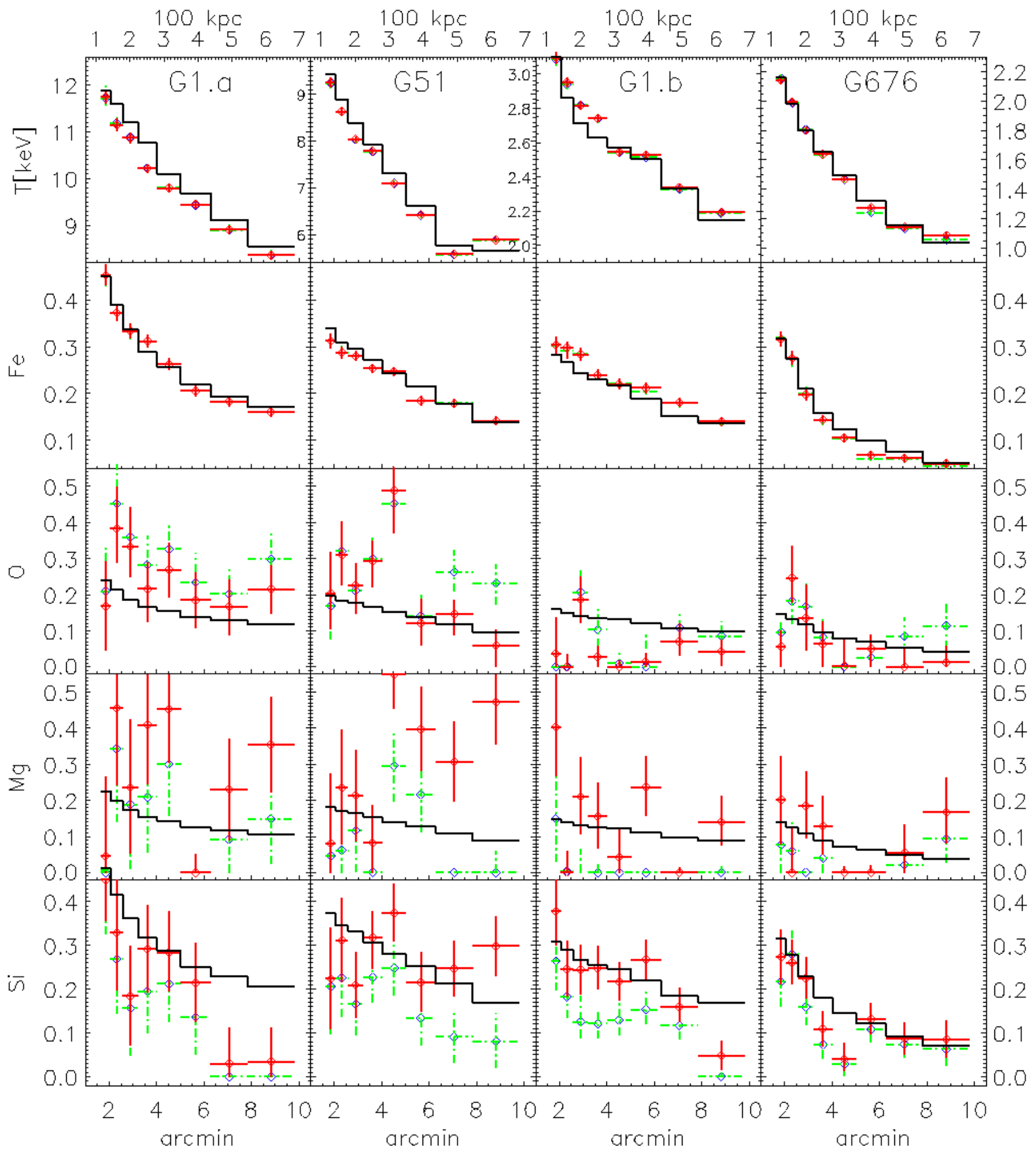

FIG. 4.- Comparison of the spectroscopic and projected input radial profiles. From top to bottom we show the Temperature, Iron, Oxygen, Magnesium, Silicon profiles, respectively. Each column correspond to a different mock cluster (from left right to right G1.a, G51, G1.b and G676 clusters). Solid black line represents the spectroscopic-like temperature and the emission-weighted metal abundance. Green dashed and solid red points with relative error bars refer to the spectroscopic values derived by using Methods 1 and 2, respectively (see text). 


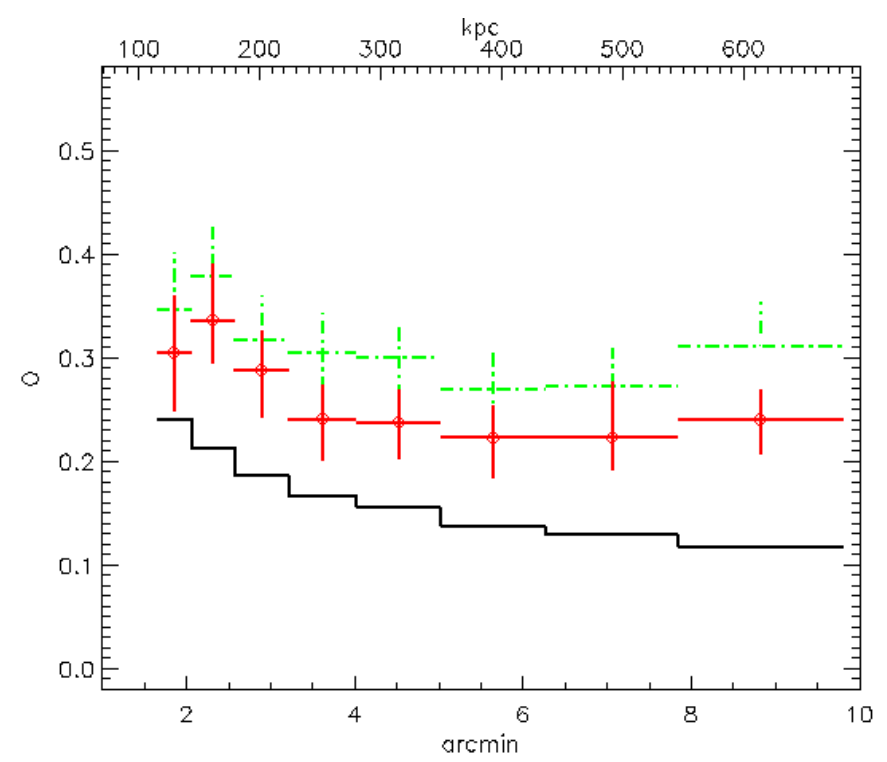

FIG. 5. - The red lines represent the spectra obtained from XSPEC reshifted at $z=0$ assuming a VMEKAL model with all the metals equal to zero but $\mathrm{O}, \mathrm{Si}, \mathrm{Mg}$ and $\mathrm{Fe}$ which are fixed to the solar values: the temperature is varying from 1, 2, 3 (top panels starting from the left) to 5, 8, $10 \mathrm{keV}$ (bottom panels). The separated contributions of the different elements are also plotted: Oxygen, Magnesium, Silicon and Iron are shown by green, blue, cyan and black to lines. All spectra are convolved with the response of EPIC MOS-1 camera; very similar results can be obtained considering the EPIC MOS-2 camera.

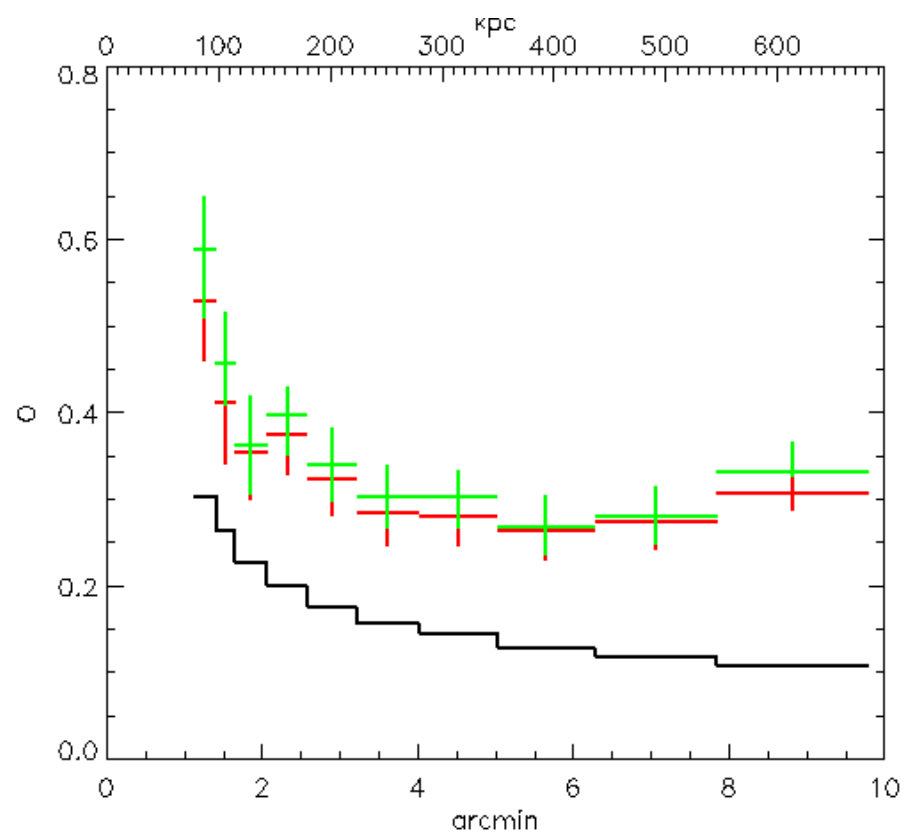

FIG. 6. - The radial profile of Oxygen in G1.a observed with an exposure time of 1 Ms. The meaning of the lines is as in Fig. 4 

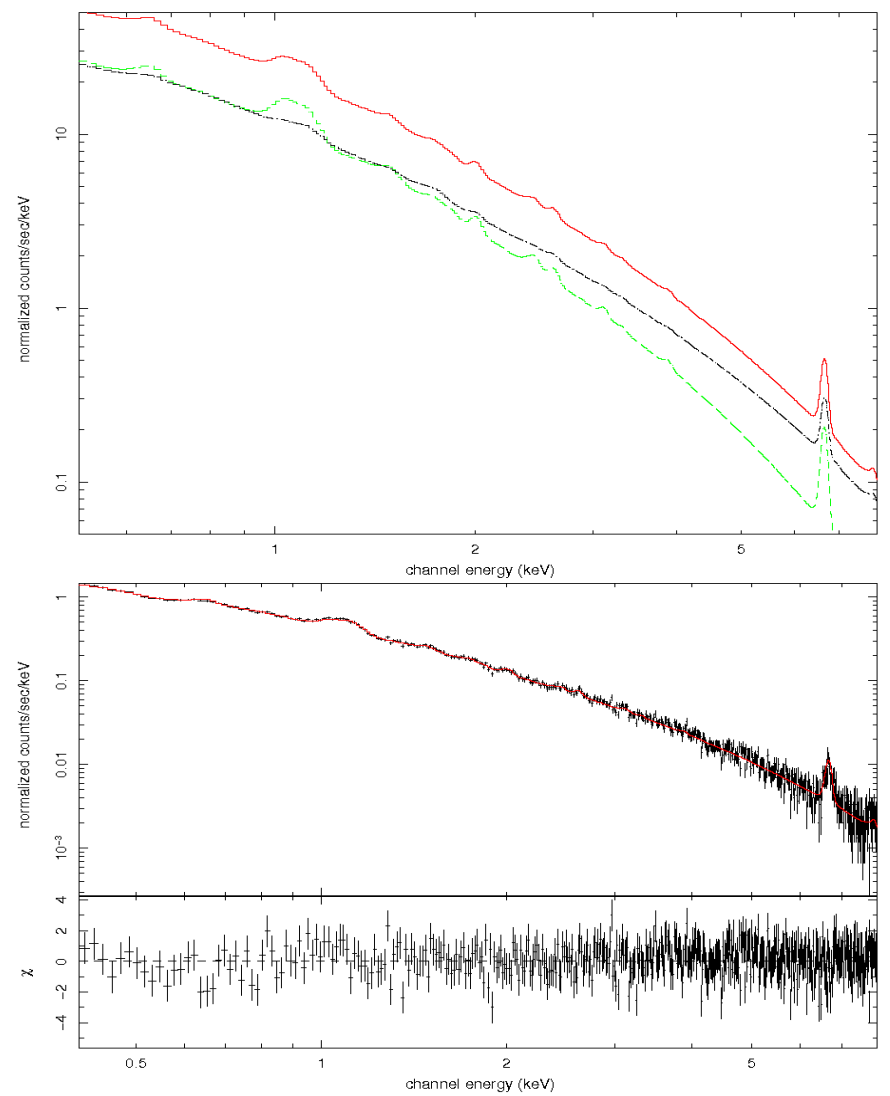

Fig. 7. - Top panel: Spectra of a plasma at $T_{1}=1 \mathrm{keV}$ (green line), $T_{2}=4 \mathrm{keV}$ (black line) and their combination (red line). Bottom panel: a fake spectrum with 450.000 counts obtained by combining a plasma with $T_{1}=2 \mathrm{keV}$ and $Z_{1}=0.2$ and a plasma with $T_{2}=3 \mathrm{keV}$ and $Z_{2}=0.1$. The red line shows the resulting best-fitting model (see text for more details). The lowest box presents the corresponding residuals.

TABLE 2

VALUES OF OXYGEN ABUNDANCES (WITH THEIR $1 \sigma$ ERROR BARS), DERIVED BY FITTING A SPECTRA, WHICH IS THE COMBINATION OF TWO PLASMAS AT 6 AND $8 \mathrm{kEV}$ (MORE DETAILS IN THE TEXT). IN THE $\chi^{2}$ FITTING PROCEDURE WE FIX THE TEMPERATURE AT THE SPECTROSCOPIC-LIKE VALUE AND THEN WE FIX ALSO THE NORMALIZATION, CONSIDERING THE ITS THEORETICAL VALUE

(2) AND SMAller VAlues $(1.995,1.990,1.985)$. The PERCENTAGEs (WITHIN BRACKETS) REPRESENT THE DIFFERENCE BETWEEN THE VALUES RECOVERED FROM THE SPECTRAL ANALYSIS AND THE EXPECTED EMISSION-WEIGHTED ONE: O=0.149.

\begin{tabular}{lcccc} 
& $N=2.000$ & 1.995 & 1.990 & 1.985 \\
\hline $\mathrm{O}_{\min }$ & 0.126 & 0.148 & $0.175(15 \%)$ & $0.192(29 \%)$ \\
$\mathrm{O}$ & 0.141 & $0.162(9 \%)$ & $0.184(23 \%)$ & $0.198(38 \%)$ \\
$\mathrm{O}_{\max }$ & 0.155 & $0.176(18 \%)$ & $0.198(33 \%)$ & $0.220(48 \%)$
\end{tabular}

$\chi_{\text {Red }}^{2}(70$ d.o.f. $) \quad 1.08 \quad 1.08 \quad 1.12 \quad 1.20$



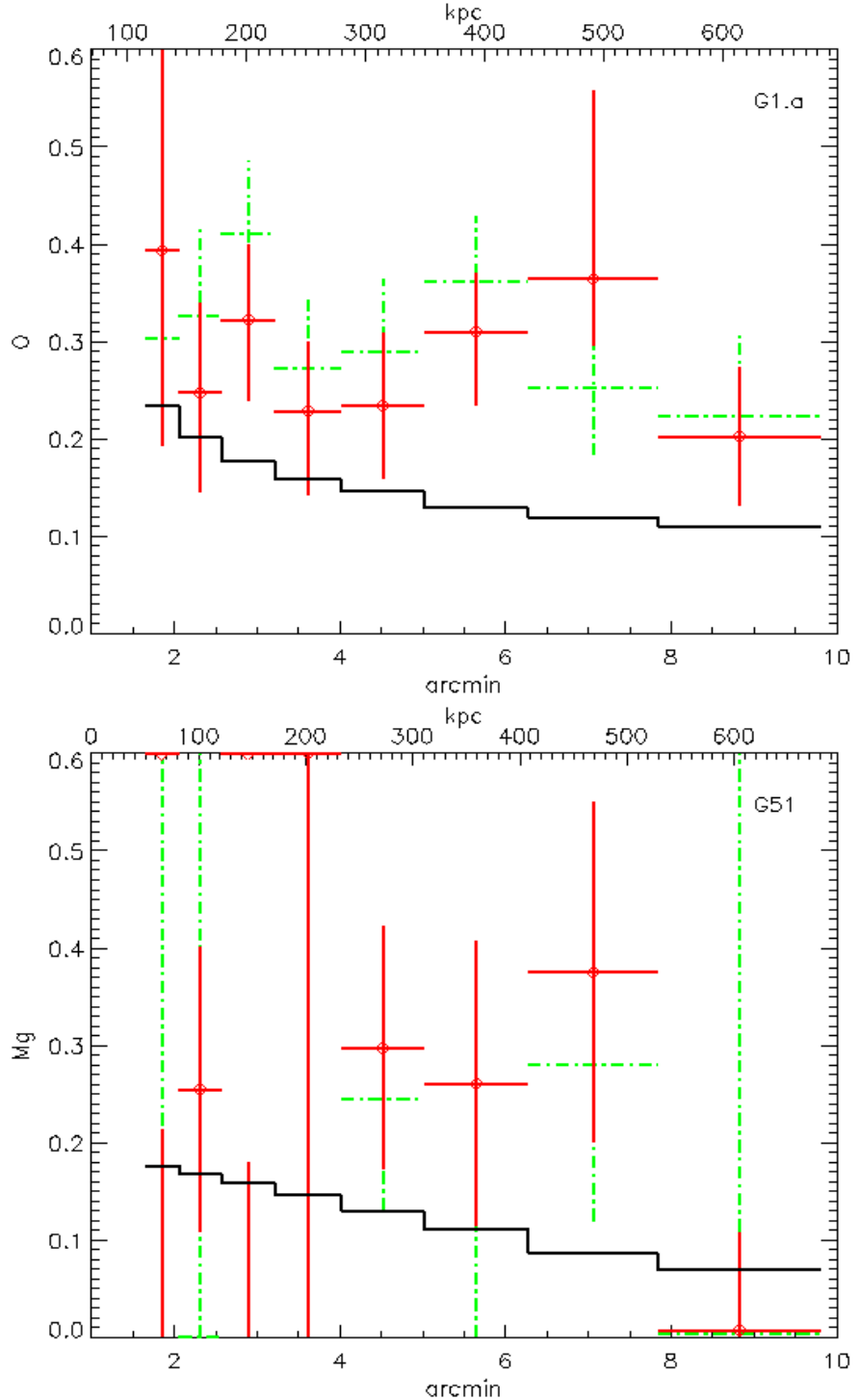

FIG. 8.- The radial profiles for Oxygen in G1.a (top panel) and Magnesium in G51 (bottom panel), obtained considering only particles with temperature larger than $5 \mathrm{keV}$. Symbols and lines are as in Fig. 4

TABLE 3

VALUES OF TEMPERATURE AND IRON ABUNDANCES (WITH THEIR $1 \sigma$ ERROR BARS), DERIVED By FITTING A SPECTRA OF A T=2

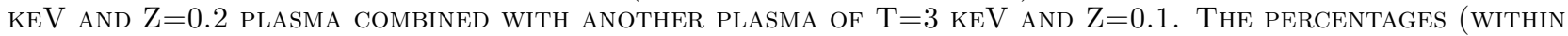
BRACKETS) REPRESENT THE DIFFERENCE BETWEEN THE VALUES RECOVERED FROM THE SPECTRAL ANALYSIS AND THE EXPECTED EMISSION-Weighted ONE: FE=0.144. The EXPECTED SPECTROSCOPIC-LIKE VAlue OF THE TEMPERATURE IS EQUAL TO 2.45.

\begin{tabular}{lcccccc} 
\# counts & $4.510^{3}$ & $910^{3}$ & $4.510^{4}$ & $910^{4}$ & $4.510^{5}$ & $910^{5}$ \\
\hline $\mathrm{T}_{\min }$ & 2.361 & 2.380 & 2.484 & 2.426 & 2.459 & 2.448 \\
$\mathrm{~T}$ & 2.439 & 2.434 & 2.510 & 2.444 & 2.470 & 2.454 \\
$\mathrm{~T}_{\max }$ & 2.520 & 2.490 & 2.536 & 2.462 & 2.476 & 2.460 \\
& & & & & & \\
$\mathrm{Fe}_{\min }$ & $0.158(10 \%)$ & $0.158(10 \%)$ & $0.172(19 \%)$ & $0.174(21 \%)$ & $0.170(18 \%)$ & $0.162(12 \%)$ \\
$\mathrm{Fe}$ & $0.199(38 \%)$ & $0.186(29 \%)$ & $0.185(28 \%)$ & $0.183(27 \%)$ & $0.174(21 \%)$ & $0.165(16 \%)$ \\
$\mathrm{Fe}_{\max }$ & $0.243(69 \%)$ & $0.215(49 \%)$ & $0.198(37 \%)$ & $0.192(33 \%)$ & $0.178(24 \%)$ & $0.168(17 \%)$
\end{tabular}

